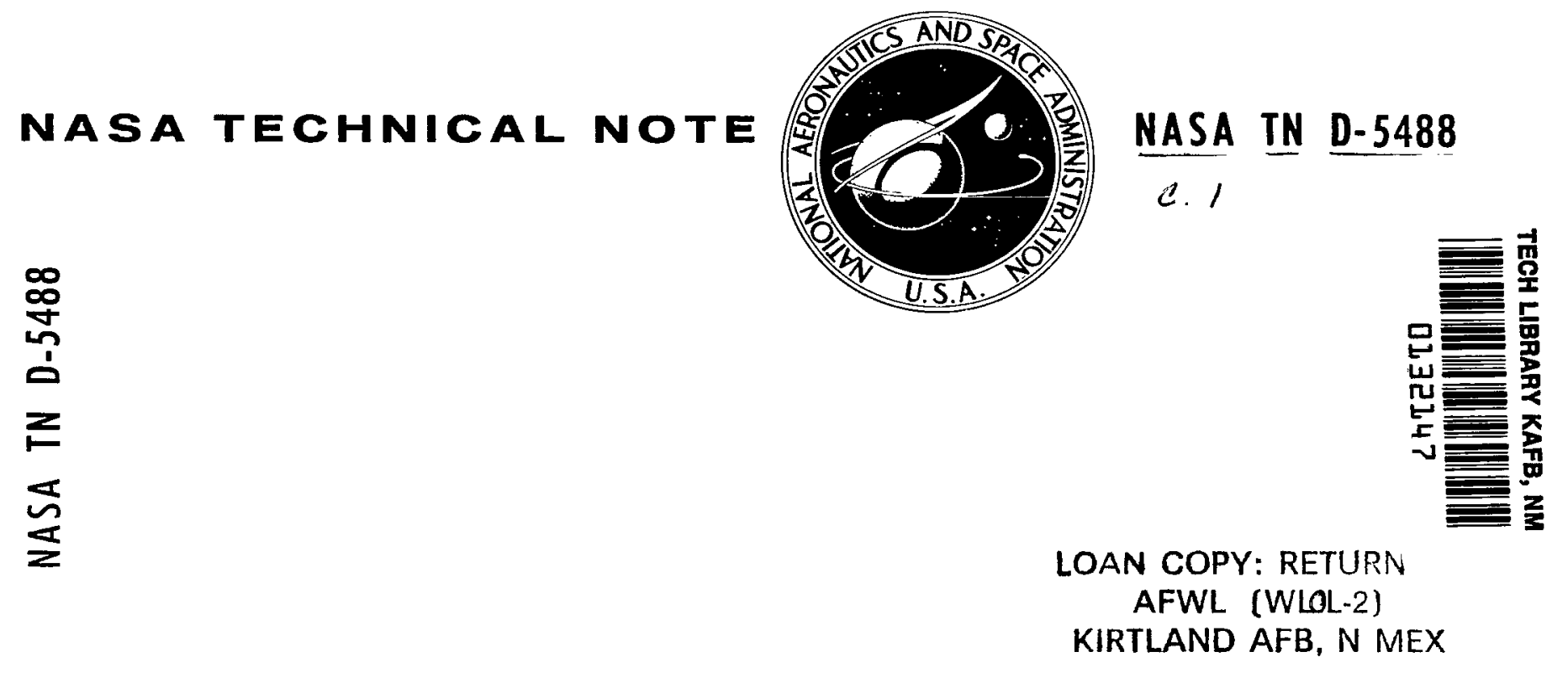

\title{
MAGNETOTHERMAL OSCILLATIONS IN PRESSURE-ANNEALED, PYROLYTIC GRAPHITE
}

by Dennis J. Flood

Lewis Research Center Cleveland, Obio 
1. Report No. NASA TN D-5488

2. Government Accession No.

4. Title and Subtitle MAGNETOTHERMAL OSCILLATIONS IN PRESSUREANNE ALED, PYROLYTIC GRAPHITE

7. Author(s) by Dennis J. Flood

9. Performing Organization Name and Address Lewis Research Center National Aeronautics and Space Administration Cleveland, Ohio 44135

12. Sponsoring Agency Name and Address

National Aeronautics and Space Administration Washington, D.C. 20546

15. Supplementary Notes

16. Abstract

Magnetothermal oscillations arising from the majority hole carriers have been observed in pressure-annealed, pyrolytic graphite. The period of the oscillations was measured as a function of angle between the magnetic field $\mathrm{H}$ and the c-axis of the crystal. The period for $\mathrm{H}$ along the c-axis is $0.151_{ \pm} 0.005$ tes $^{-1}$. The sample had a resistance ratio of $1 / 2$ from room temperature to $4.2 \mathrm{~K}$. Data were obtained at $1.3 \mathrm{~K}$ in fields ranging from 0.35 to 10 tesla.
17. Key Words (Sugiested by Author(s)) magnetothermal effects Fermi surfaces graphite
20. Security Classif. (of this page)
Unclassified

20. Security Classif. (of this page)
Unclassified
18. Distribution Statement

Unclassified - unlimited
19. Security Classif. (of this report) Unclassified
21. No. of Pages
23

22. Price* $\$ 3.00$

*For sale by the Clearinghouse for Federal Scientific and Technical Information Springfield, Virginia 22151 


\title{
MAGNETOTHERMAL OSCILLATIONS IN PRESSURE-
}

\author{
ANNEALED, PYROLYTIC GRAPHITE \\ by Dennis J. Flood \\ Lewis Research Center
}

SUMMARY

Magnetothermal oscillations have been observed for the first time in a sample of pressure-annealed pyrolytic graphite. Changes in sample temperature of the order of $5 \times 10^{-5} \mathrm{~K}$ were measured using a field modulation technique. The period was studied as a function of the angle between the c-axis of the crystal and the magnetic field direction. Two periods were observed whose values for magnetic-field strength $H$ parallel to the c-axis are $0.151 \pm 0.005$ and $0.85 \pm 0.20$ tesla $^{-1}$. The former agrees well with the period of the majority hole carriers obtained by other workers using different techniques in both single crystal and pyrolytic graphite. The second period is tentatively ascribed to the minority electrons and is lower than the values previously reported

The measurements reported here have extended the range of magnetic field over which quantum oscillations in any equilibrium phenomenon have been observed in pyrolytic graphite. The phase factor $\gamma$ has been determined, with the use of the high magnetic field data, to be $0.50 \pm 0.10$.

\section{INTRODUCTION}

The term "magnetothermal oscillation" refers to the oscillatory dependence of the temperature of a thermally isolated sample on inverse magnetic field. Kunzler, Hsu, and Boyle (ref. 1) originally observed the phenomenon in a single crystal of bismuth by a simple dc technique. Following that, LePage, Garber, and Blatt (ref. 2), using a variation of the dc technique of Kunzler et al., reported the effect in beryllium. McComb and Seidel (refs. 3 to 5 ), using a field modulation technique, investigated the effect in bismuth, antimony, and beryllium.

This report discusses the observation of magnetothermal oscillations in pressureannealed, pyrolytic graphite by means of both a field modulation technique and the modi- 
fied dc technique of LePage et al. The oscillations were studied as a function of orientation in fields ranging from 0.35 tesla to 10 tesla at temperatures between 1.3 and $1.5 \mathrm{~K}$.

Pyrolytic graphite is essentially a polycrystalline material which exhibits a high degree of order in one direction, and nearly complete disorder in the plane perpendicular to that direction. The behavior of the electronic properties of pyrolytic graphite can qualitatively approach that of the electronic properties of single crystal graphite as the order is improved through the high-pressure annealing process (refs.6 and 7). Williamson, Foner, and Dresselhaus (ref. 8) have observed deHaas-van Alphen oscillations in pyrolytic graphite, and compared the results with those obtained from deHaasvan Alphen measurements in single crystal graphite by Soule (ref. 9) and with magnetoresistance measurements (Shubnikov - de Haas effect) in single crystal graphite by Soule, McClure, and Smith (ref. 10). They found that the general features of the majority carrier pockets are identical for single crystal and pyrolytic graphite, but that details for the minority carrier pockets near the zone corners differ somewhat. Their conclusion was that the Slonczewski-Weiss band model for graphite (ref. 11) is applicable to pyrolytic graphite, and that the electron and hole Fermi surfaces are closed.

\section{THEORETICAL}

\section{Magnetothermal Oscillations}

According to thermodynamics, the change in internal energy $U$ of a homogeneous system in which temperature $\mathrm{T}$, magnetization $\mathrm{M}$, and magnetic field strength $\mathrm{H}$ are the only state variables of interest is given by

$$
d U=d^{\prime} Q-d^{\prime} W=C_{H} d T+T\left(\frac{\partial M}{\partial T}\right)_{H} d H
$$

where $d^{\prime} Q$ is positive for heat flow into the system, $d^{\prime} W$ is positive for work done by the system, and $\mathrm{C}_{\mathrm{H}}$ is the total heat capacity at constant magnetic field. The rate at which heat flows from the system into a reservoir is

$$
\frac{\mathrm{d}^{\prime} \mathrm{Q}}{\mathrm{dt}}=-\kappa\left(\mathrm{T}_{\mathrm{S}}-\mathrm{T}_{\mathrm{r}}\right)=-\kappa \Delta \mathrm{T}
$$

where $T_{S}$ is the system temperature, $T_{r}$ is the temperature of the reservoir, and $\kappa$ is simply $\mathrm{Ka} / \mathrm{L}$, where $\mathrm{K}$ is the thermal conductivity of the path of length $\mathrm{L}$ and crosssectional area a which connects the system to the reservoir. From equations (1) 
and (2), the temperature difference between the sample and the reservoir $\Delta \mathrm{T}$, is given by

$$
-\kappa \Delta T=C_{H} \frac{d T_{S}}{d t}+T_{S}\left(\frac{\partial M}{\partial T}\right)_{H} \frac{d H}{d t}+\frac{d^{\prime} W}{d t}
$$

The magnetization of a system of conduction electrons contained within a solid has been derived by Lifshitz and Kosevich (ref. 12). Their original calculation has since been refined by several authors (refs. 13 to 15 ). The oscillatory part of $M$, which is all that is of interest here, is given by

$$
\mathrm{M}_{\mathrm{osc}}=\frac{2 \pi \mathrm{f}}{\mathrm{H}^{1 / 2}} \mathrm{~T} \sum_{l=1}^{\infty} \frac{\mathrm{P}_{l} \exp \left(\frac{-l \mathrm{x}}{\mathrm{T}_{\mathrm{H}}}\right)}{l^{1 / 2} \sinh \frac{l \mathrm{~T}}{\mathrm{~T}_{\mathrm{H}}}} \sin \left(\frac{2 \pi l \mathrm{f}}{\mathrm{H}}-2 \pi l \gamma \mp \frac{\pi}{4}\right)
$$

where

$$
\begin{gathered}
\mathrm{P}_{l}=-2 \mathrm{Vk}_{\mathrm{B}}\left(\frac{\mathrm{e}}{\mathrm{hc}}\right)^{3 / 2}\left[\frac{\partial^{2} \mathrm{~A}\left(\mathrm{E}_{\mathrm{F}}\right)}{\partial \mathrm{k}_{\mathrm{z}}^{2}}\right]_{\mathrm{k}_{\mathrm{O}}}^{-1 / 2} \cos \frac{\pi l \mathrm{gm}^{*}}{2 \mathrm{~m}_{\mathrm{o}}} \\
\mathrm{T}_{\mathrm{H}}=\frac{\mathrm{e \hbar H}}{2 \pi^{2} \mathrm{~m}^{*} \mathrm{ck}_{\mathrm{B}}}
\end{gathered}
$$

Dingle temperature:

$$
\mathbf{x}=\frac{\hbar}{\pi \mathrm{k}_{\mathrm{B}} \tau_{\mathrm{D}}}
$$

de Haas - van Alphen frequency:

$$
f=\frac{c \hbar}{2 \pi \mathrm{e}} \mathrm{A}_{\mathrm{o}}\left(\mathrm{E}_{\mathrm{F}}\right)
$$

$\mathrm{k}_{\mathrm{o}}$ defines the location in $\mathrm{k}$-space of the extremal area denoted by $\mathrm{A}_{\mathrm{o}}\left(\mathrm{E}_{\mathrm{F}}\right), \mathrm{E}_{\mathrm{F}}$ is the Fermi energy, $\mathrm{m}^{*}$ is the effective mass given by $\left(\hbar^{2} / 2 \pi\right) \partial \mathrm{A}\left(\mathrm{E}_{\mathrm{F}}\right) / \partial \mathrm{E}_{\mathrm{F}}, \mathrm{V}$ is the volume of the sample, $g$ is the spin-splitting factor (ref. 13), $\tau_{D}$ (ref. 14) is the average time an electron travels between collisions, and $\mathrm{k}_{\mathrm{B}}$ is the Boltzmann constant. The other 
symbols are defined in the appendix. The quantity $\gamma$ which appears in the argument of the sine in equation (4) is the phase factor which appears in the expression developed by Onsager (ref. 16) for the quantization of the area of the orbits traveled by the electrons:

$$
A_{n}=(n+\gamma) \frac{e H}{\hbar c}
$$

where $\mathbf{n}$ is an integer quantum number which labels the energy levels (Landau levels) of the electrons in the magnetic field. For free electrons, or for electrons which obey a general quadratic dispersion law, $\gamma$ is equal to $1 / 2$. (This is true basically because the Schroedinger equation in such instances reduces to that for the harmonic oscillator, with eigenvalues $\left.E_{n}=(n+1 / 2) \hbar \omega^{*}\right)$. Chambers (ref. 17) has recently pointed out that when interband transitions can be ignored, that is, only weak fields and weak electronelectron interactions allowed, $\gamma$ is expected to be $1 / 2$ even for arbitrary Fermi surfaces.

The expression in equation (4) is subject to some limitations. The magnetic field and temperature must be such that $\mathrm{E}_{\mathrm{F}}>>\mathrm{k}_{\mathrm{B}} \mathrm{T}$ and $\left(\hbar_{\mathrm{eH}} / \mathrm{m}^{*} \mathrm{c}\right)>>\mathrm{k}_{\mathrm{B}} \mathrm{T}$. It is also necessary that the argument of the sine term in equation (4) be large, that is, that $(2 \pi f / H)>1$. The last condition means simply that the expression for $M_{o s c}$ is no longer valid in the so-called "quantum limit regime," where the Landau-level spacing becomes comparable to the Fermi energy $\mathrm{E}_{\mathrm{F}}$.

Direct substitution of equation (4) into equation (3) results in

$$
\begin{aligned}
-\kappa \Delta \mathrm{T}=\mathrm{C}_{\mathrm{H}, \theta} \frac{\mathrm{dT}_{\mathrm{S}}}{\mathrm{dt}}+\mathrm{T}_{\mathrm{S}} & {\left[\frac{2 \pi \mathrm{f}}{\mathrm{H}^{1 / 2}} \sum_{l=1}^{\infty} l^{-1 / 2} \sin \left(\frac{2 \pi l \mathrm{f}}{\mathrm{H}}-2 \pi l \gamma \mp \frac{\pi}{4}\right)\right.} \\
& \left.\times\left(1-\frac{l \mathrm{~T}_{\mathrm{S}}}{\mathrm{T}_{\mathrm{H}}} \operatorname{coth} \frac{l \mathrm{~T}_{\mathrm{S}}}{\mathrm{T}_{\mathrm{H}}}\right) \frac{\mathrm{P}_{l} \exp \left(\frac{-l \mathrm{x}}{\mathrm{T}_{\mathrm{H}}}\right)}{\sinh \left(\frac{l \mathrm{~T}}{\mathrm{~T}_{\mathrm{H}}}\right)}\right] \frac{\mathrm{dH}}{\mathrm{dt}}+\frac{\mathrm{d}^{\prime} \mathrm{W}}{\mathrm{dt}}
\end{aligned}
$$

The expected amplitude of the oscillations is at most of the order of $10^{-3} \mathrm{~K}$, and for many materials the amplitude will be less than $10^{-4} \mathrm{~K}$. For near-adiabatic conditions, the term on the left in equation (7) can be neglected, and the change in sample temperature is given by 


$$
\begin{aligned}
\mathrm{dT}_{\mathrm{S}} \simeq \frac{-\mathrm{T}_{\mathrm{S}}}{\mathrm{C}_{\mathrm{H}, \theta}}\left[\frac{2 \pi \mathrm{f}}{\mathrm{H}^{1 / 2} \sum_{l=1}^{\infty} l^{-1 / 2} \sin \left(\frac{2 \pi l \mathrm{f}}{\mathrm{H}}-2 \pi l \gamma \mp \frac{\pi}{4}\right)}\right. \\
\left.\quad \times\left(1-\frac{l \mathrm{~T}_{\mathrm{S}}}{\mathrm{T}_{\mathrm{H}}} \operatorname{coth} \frac{l \mathrm{~T}_{\mathrm{S}}}{\mathrm{T}_{\mathrm{H}}}\right) \frac{\mathrm{P}_{l} \exp \left(\frac{-l \mathrm{x}}{\mathrm{T}_{\mathrm{H}}}\right)}{\sinh \frac{l \mathrm{~T}_{\mathrm{S}}}{\mathrm{T}_{\mathrm{H}}}}\right] \mathrm{dH}-\mathrm{d}^{\prime} \mathrm{W}
\end{aligned}
$$

The last term on the right in equation (8) includes all the dissipative effects acting to change the temperature of the system such as joule heating in the thermometers, eddy currents, and so on.

\section{Band Theory of Graphite}

The crystal structure of graphite is well known and is illustrated in figure 1 . The lattice is hexagonal close-packed, and the hexagonal Brillouin zone corresponding to it is shown in figure 2. Only a small fraction of the zone is occupied by the current carriers, so that only the energy bands near the vertical edges of the zone are important for the determination of transport effects. Accordingly, Slonczewski and Weiss (ref. 11) have derived an effective-mass Hamiltonian for the energy bands near the vertical edges. Their original calculations were developed further by McClure (ref. 18), who included the effects

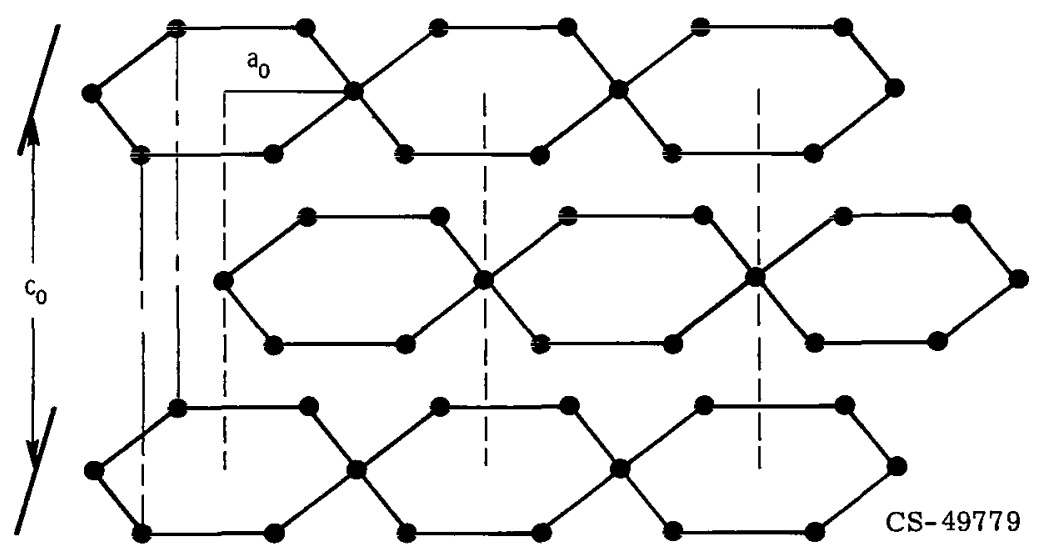

Figure 1. - Crystal structure of graphite. Centers of hexagons in one layer lie over corners of hexagons in layer beneath it. Unit cell parameters are $a_{0}=2.456 \AA$ and $c_{0}=6.712 \AA$. 


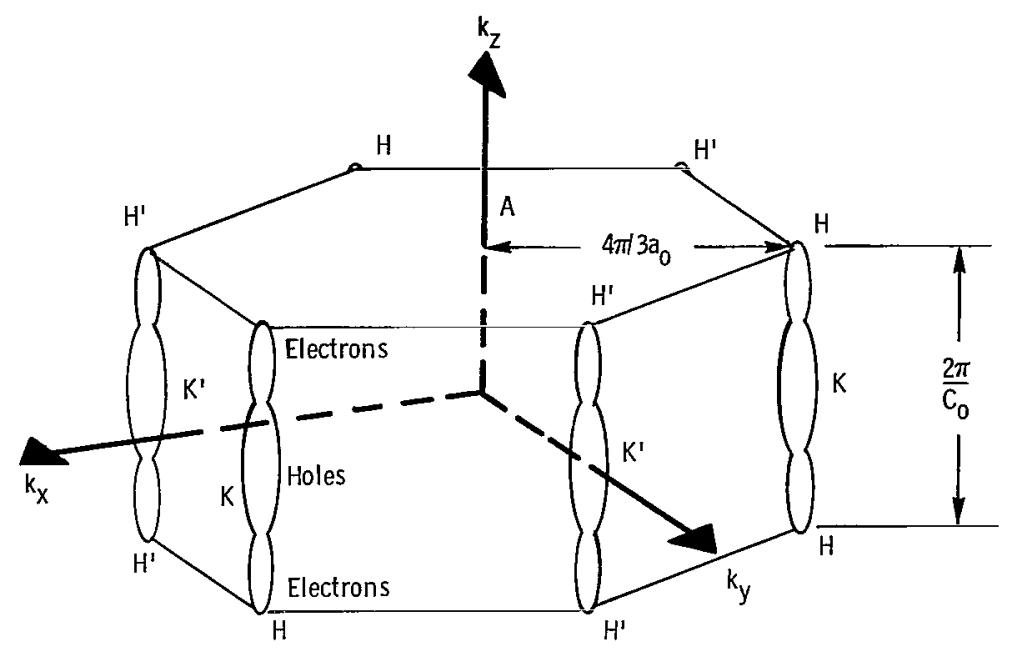

Figure 2. - Brillouin zone of graphite (from Williamson et al. (ref. 15)).

of a magnetic field. The energy bands derived by the former are shown in figure 3 , which indicates a hole pocket centered at $\mathrm{k}_{\mathrm{z}}=0$, and two electron pockets with extremal areas near $c_{0} k_{z} / 2 \pi= \pm 0.35$, where $c_{0}$ is twice the spacing between layers of the crystal. These electron and hole majority pockets arise from the energy band labeled $\mathrm{E}_{3}$. In ad-
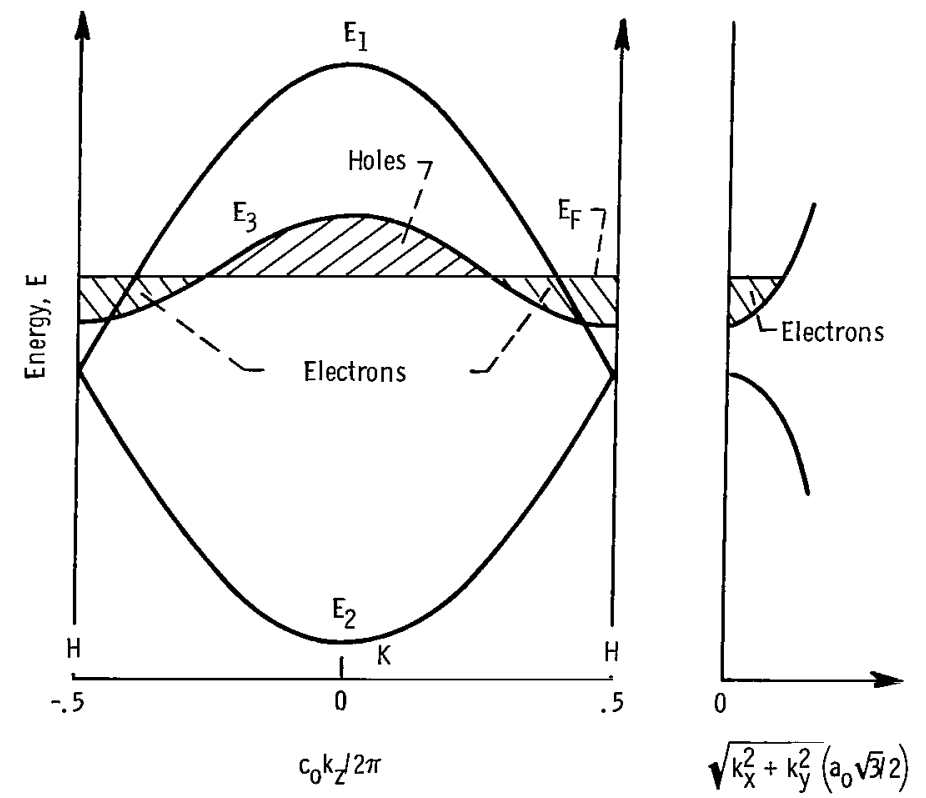

Figure 3. - Energy bands of graphite near vertical zone edge HKH at zero field as given by Slonczewski-Weiss model. Small pocket of electrons shown at right side of figure arises from $E_{1}$ band and joins smoothly with pocket of electrons formed by $E_{3}$ band (from Williamson et al. (ref. 15)). (Small pocket forms caps of ellipsoids at points $H$ in fig. 2 .) 
dition there are small minority pockets of electrons near the points $c_{o} k_{z} / 2 \pi= \pm 0.5$ which arise from the $E_{1}$ band. The minority pockets join smoothly with the electron pockets in the $\mathrm{E}_{3}$ band in the absence of a magnetic field, or when spin-orbit interactions are neglected. Figure 4(a) shows the Fermi surface in a reduced zone scheme for the case

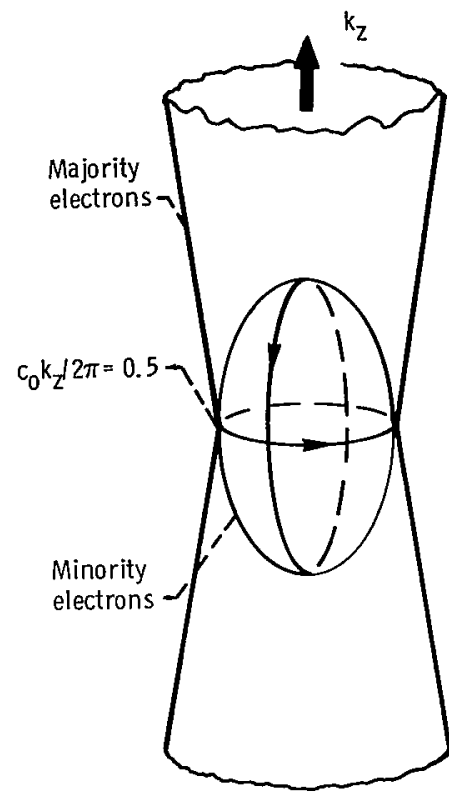

(a) Minority electron pocket at zero field, neglecting spinorbit interaction. The orbit at $\mathrm{k}_{\mathrm{z}}=\pi \mathrm{c}_{0}$ is not stationary.

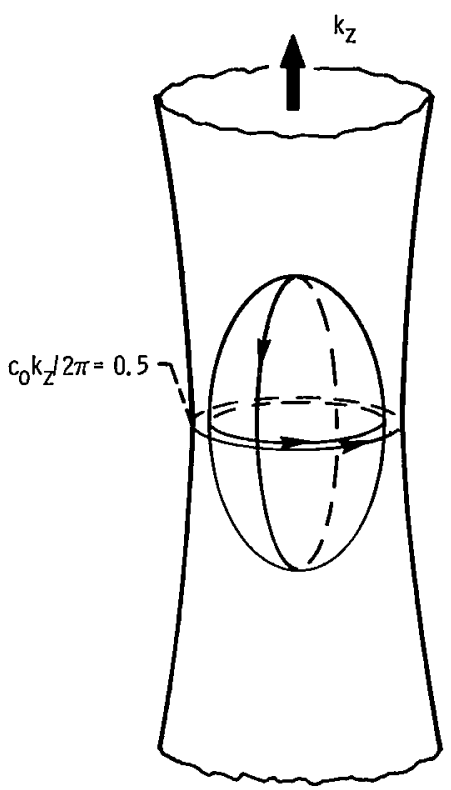

(b) Effects of spin-orbit interaction. Two stationary orbits are produced as a result of the separation of the Fermi surfaces of the two pockets.

Figure 4. - Details of Fermi surface near the Brillouin zone corners in the extended zone scheme (from Williamson et al, ref. 15).

where the two pockets are joined smoothly onto one another, and figure 4(b) shows the effects of including a spin-orbit interaction in the Hamiltonian. Only for the second case does a true extremal deHaas - van Alphen orbit exist. (McClure (ref. 18), however, has shown that the magnetic energy levels, even in the absence of a spin-orbit interaction, exhibit stationary points at the same values of $\mathrm{k}_{\mathrm{z}}$ as in the preceeding discussion.) This picture may not be complete. Soule (ref. 9) has suggested, on the basis of acceptorimpurity doping studies in single crystal graphite that there may be some minority hole pockets as well. These may be located around the majority hole pocket as shown in figure 5 . 


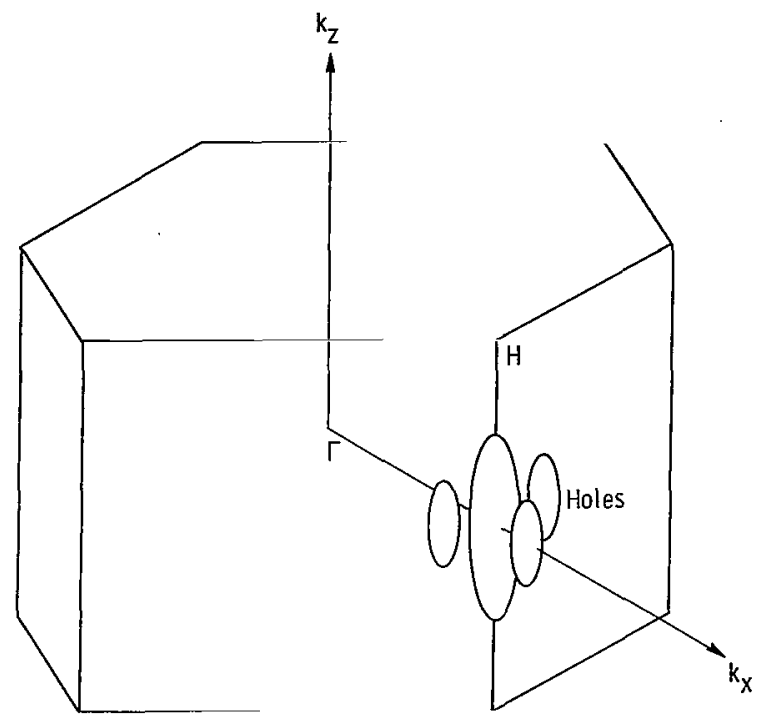

Figure 5. - Brillouin zone with possible location of minority hole "outrigger" Fermi surfaces as reduced about zone edge (from Soule, ref. 9).

\section{EXPERIMENTAL}

\section{Experimental Techniques}

There are basically two ways to detect magnetothermal oscillations: resistance bridge thermometry (refs. 1 and 2), and a field modulation technique (refs. 3 to 5 ). Figure 6 contains a schematic diagram of the experimental setup for the former. A lowfrequency ac resistance bridge is used to detect the change in resistance of a carbon thermometer attached to the sample. The bridge input voltage is supplied by the refer-

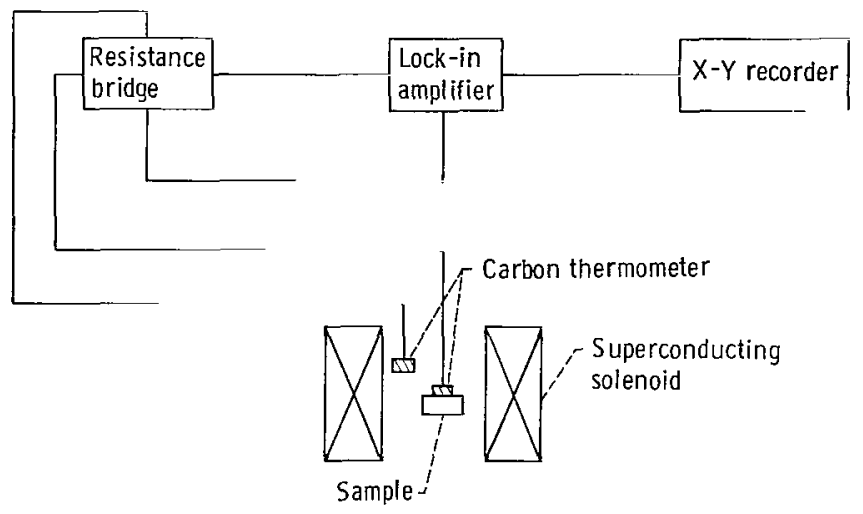

Figure 6. - Schematic diagram of bridge technique. 
ence oscillator of a lock-in amplifier. The output of the bridge is then synchronously detected by the lock-in amplifier and converted to a dc voltage which is fed to an X-Y recorder. This technique requires a very careful adjustment of heat leaks from the sample to the bath so that eddy current heating in the sample will not raise its average temperature appreciably. It is also necessary, however, to provide a long enough thermal time constant so that the sample can vary its temperature at least enough to be observed. Thermometer magnetoresistance can also affect the measurements in the form of a large monotonic background. Nonetheless, in particular situations the technique is quite useful and can produce calibrated amplitudes for the oscillatory temperature changes.

A schematic diagram for the field modulation technique is shown in figure 7. A small-amplitude, low-frequency, modulating magnetic field is superimposed on the monotonically swept dc field. The resulting ac component of the temperature change is measured by a lock-in amplifier. In this case the time constant from sample to bath must only be long compared with the period of the modulating field, or whatever harmonic of it which is being detected, and can be made short enough so that the sample remains at an average temperature very close to that of the bath. It is necessary, however, for the thermal relaxation time between thermometer and sample to be short compared with the period of the modulating signal so that the thermometer will follow the sample temperature at the detection frequency. This is usually not a difficult requirement to satisfy. Changes in average sample temperature which arise from eddy currents induced by the slowly swept dc field will therefore be greatly reduced under these conditions. Moreover, eddy currents induced by the modulating field will cause heating effects primarily at twice the frequency of the ac field and are eliminated by detection at the fundamental.

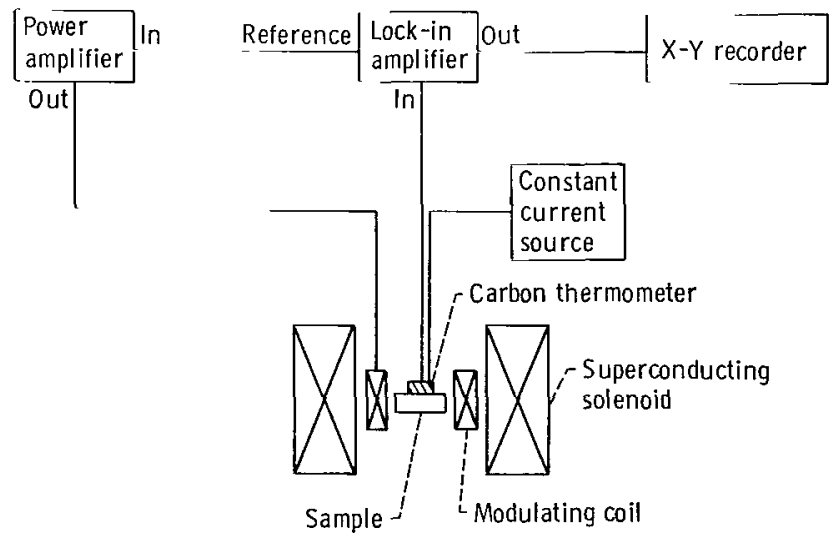

Figure 7. - Schematic diagram of field modulation technique. 


\section{Sample and Apparatus}

The sample was a rectangular rod 3.18 by 3.18 by 12 millimeters $(1 / 8$ by $1 / 8$ by $1 / 2$ in.) of pyrolytic graphite and was cut with its long dimension perpendicular to the c-axis. It had a resistance ratio $R_{300} / R_{4.2}$ of $1 / 2$ for current flow in the basal plane.

The sample holder is shown in figure 8. The spiral gear was machined such that a complete rotation by it of $360^{\circ}$ caused the gear on the sample bed to advance by one tooth, which in this case corresponded to $10^{\circ}$. The drive shaft on the spiral gear was a length of stainless-steel tubing which was sealed at the top end, fed through the vacuum line, and bolted to a brass rod at the bottom. The brass rod passed through the top of the sample chamber, which acted as a heat sink. The spiral gear was fastened in place at the bottom of the brass rod with a setscrew, and its position could be adjusted to obtain a minimum amount of backlash. The apparatus provides one degree of freedom in an external magnetic field.

The open table on which the sample was mounted was machined from oxygen-free high-conductivity copper and was supported by bushings of the same material which were soldered into the brass wall of the chamber. Wires to the sample could be clamped to the edges of the table so that rotating the holder would not cause the leads to pull directly on the sample. The clamp also provided a second heat sink for the leads in addition to one located on the wall of the chamber. The sample was secured to the table with magnetwire varnish and a second clamp. The thermometer was glued on the end of the sample away from the clamp with varnish and was electrically insulated from the sample by a piece of cigarette paper.

The dc magnetic field was provided by a 6.35 -centimeter $\left(2 \frac{1}{2}\right.$-in.) bore, 10.5 -tesla superconducting solenoid. The field was measured by a copper magnetoresistor which had been calibrated by nuclear magnetic resonance at several values of field strength. The estimated error in the calibration is \pm 0.2 percent. The modulating field was provided by a coil wrapped around the outside of the vacuum chamber containing the sample. Modulating fields up to about 0.01 tesla were obtainable.

An insert Dewar with a 5.33-centimeter (2.1-in.) inner-diameter tail was used to reach temperatures below $4.2 \mathrm{~K}$. Temperatures near $1 \mathrm{~K}$ could be attained when no power was supplied to the modulation coils. Measurements were typically made in the vicinity of $1.3 \mathrm{~K}$.

\section{Experimental Procedure}

Although both methods were tried, the field modulation technique was used almost exclusively. The primary difficulty with the bridge technique is the large monotonic back- 


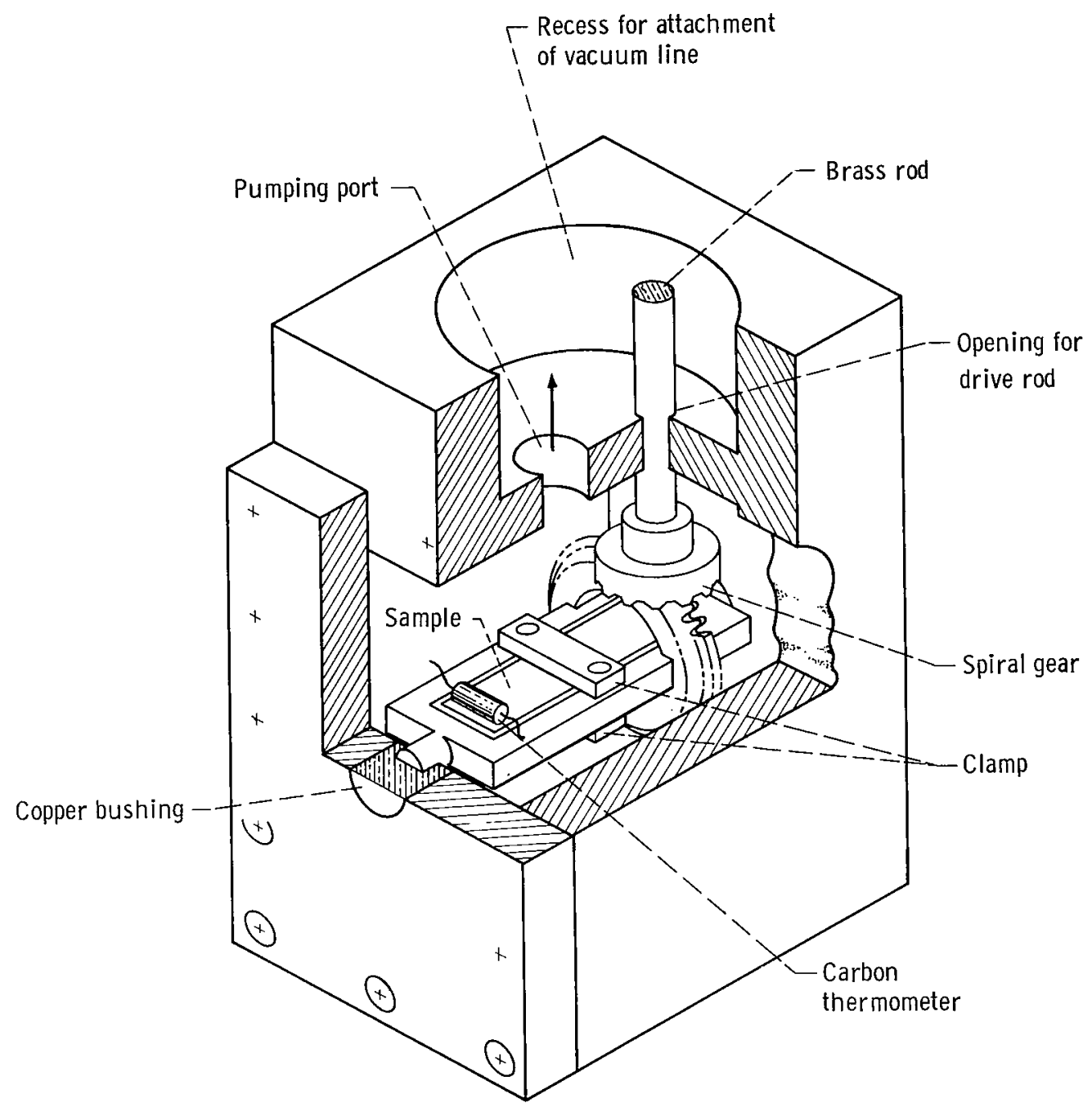

Figure 8. - Sample holder detail. 
ground arising from the thermometer magnetoresistance. This can be reduced by comparing two resistors with each other, but the necessary compensation is difficult to achieve because of differing field and temperature characteristics. This problem is not encountered with field modulation, since to a first approximation

$$
\frac{\Delta R}{\Delta \mathrm{H}} \simeq\left(\frac{\partial \mathrm{R}}{\partial \mathrm{T}}\right)_{\mathrm{H}}\left(\frac{\partial \mathrm{T}}{\partial \mathrm{H}}\right)_{\mathrm{S}}+\left(\frac{\partial \mathrm{R}}{\partial \mathrm{H}}\right)_{\mathrm{T}}
$$

The second term is very nearly constant over the entire field range and provides only an offset from zero, not an increasing background. Figure 9 is a plot of resistance as a

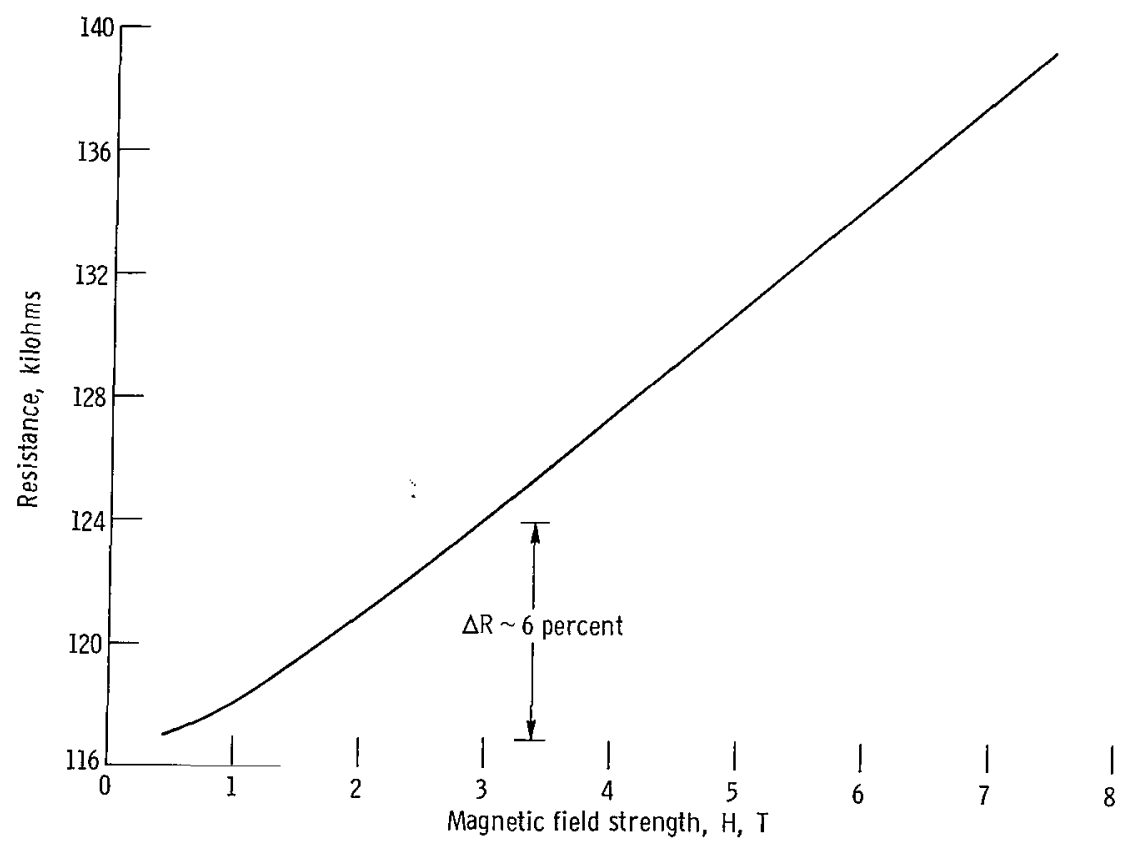

Figure 9. - Thermometer magnetoresistance at $I K$.

function of magnetic field strength under isothermal conditions and shows that $(\partial \mathrm{R} / \partial \mathrm{H})_{\mathrm{T}=1 \mathrm{~K}}$ is relatively small and does not change much over the field range used. The variation of $R$ with temperature for the same 100-ohm (nominal room temperature value), 1/8-watt resistor is shown in figure 10 . In the region around $1.3 \mathrm{~K},(\partial \mathrm{R} / \partial \mathrm{T})_{\mathrm{H}}$ is very large, and for small changes in temperature, nearly constant. Hence, changes in $\Delta \mathrm{R} / \Delta \mathrm{H}$ are the result almost entirely of changes in $(\partial \mathrm{T} / \partial \mathrm{H})_{\mathrm{S}}$.

The thermal response of the system was such that temperature changes could be detected at frequencies up to $33 \mathrm{hertz}$. Amplitudes at the higher frequencies were reduced considerably. Typically, measurements were made at 4 hertz. The integrator time con- 


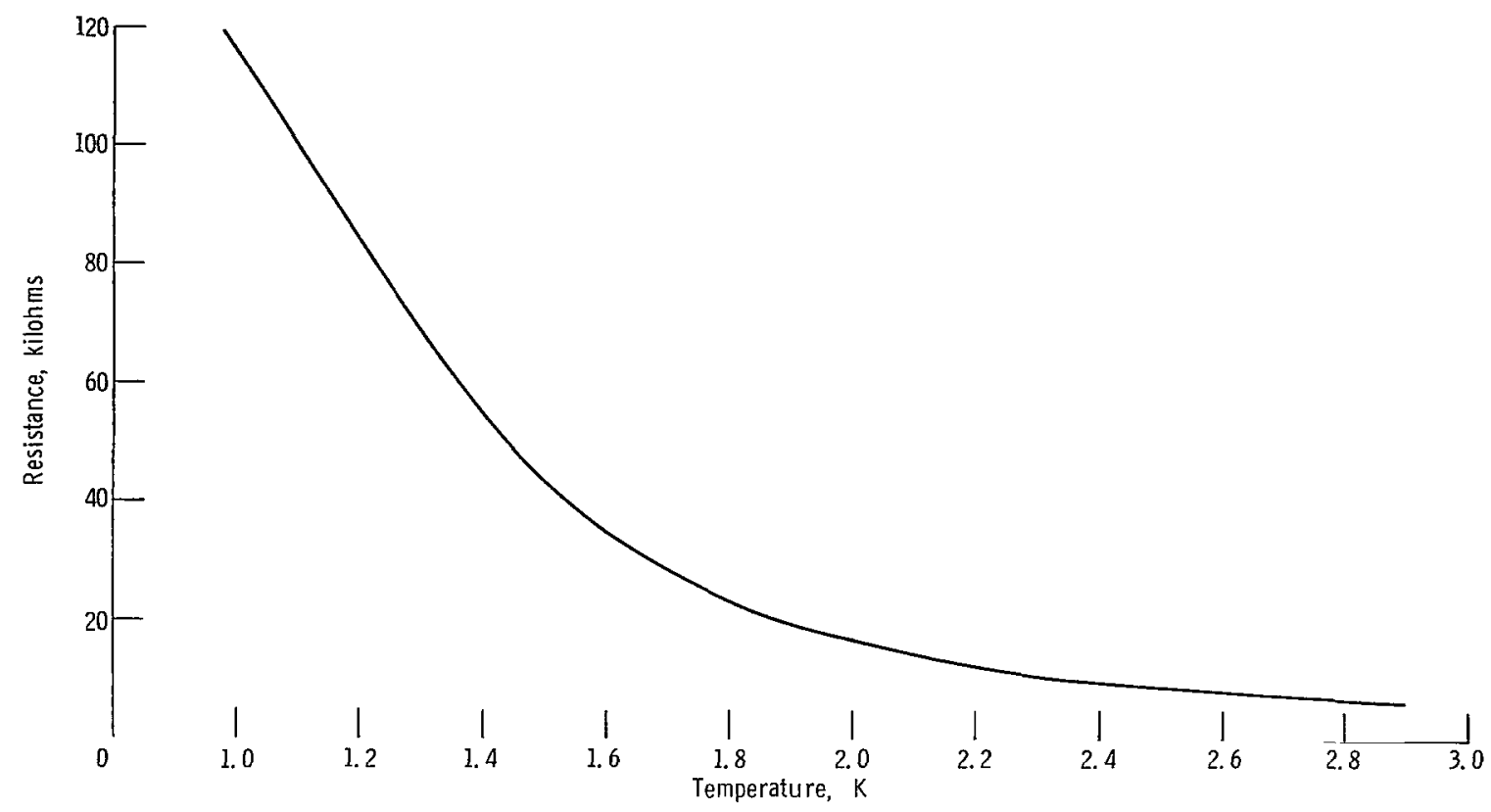

Figure 10. - Thermometer resistance as function of temperature.

stant at the output of the lock-in amplifier was usually 3 seconds. (As mentioned earlier, the frequency response is limited by the thermometer-sample contact. After the present data were collected, attempts to improve the response were partly successful, enabling data to be taken at $33 \mathrm{~Hz}$ with almost the same sensitivity as before.)

The oscillations were studied as a function of angle between $H$ and the c-axis of the crystal by manually rotating the sample holder to the desired position and then sweeping the magnetic field. The angular position of the sample was recorded by measuring the voltage across a 10-turn potentiometer. The initial error in position was estimated to be ${ }_{ \pm} 1^{\circ}$, and the relative error of any setting was \pm 0.5 percent.

A dc current of the order of 1 microampere was passed through the thermometer, and the voltage changes measured were in the range from 1 to 20 microvolts. Thermometer sensitivity was typically 0.2 volt per degree kelvin for the operating points chosen (i.e., $\mathrm{T} \simeq 1.3-1.5 \mathrm{~K}$ ).

\section{Results and Discussion}

The angular variation of the period of the observed oscillations is shown in figure 11. The value for $\mathrm{H}$ parallel to the c-axis is $0.151_{ \pm} 0.005 \mathrm{tesla}^{-1}$. This is in good agreement with the hole period obtained by Soule et al. (ref. 10) from Shubnikov - de Haas measurements in single crystal graphite, and with that obtained by Williamson et al. 


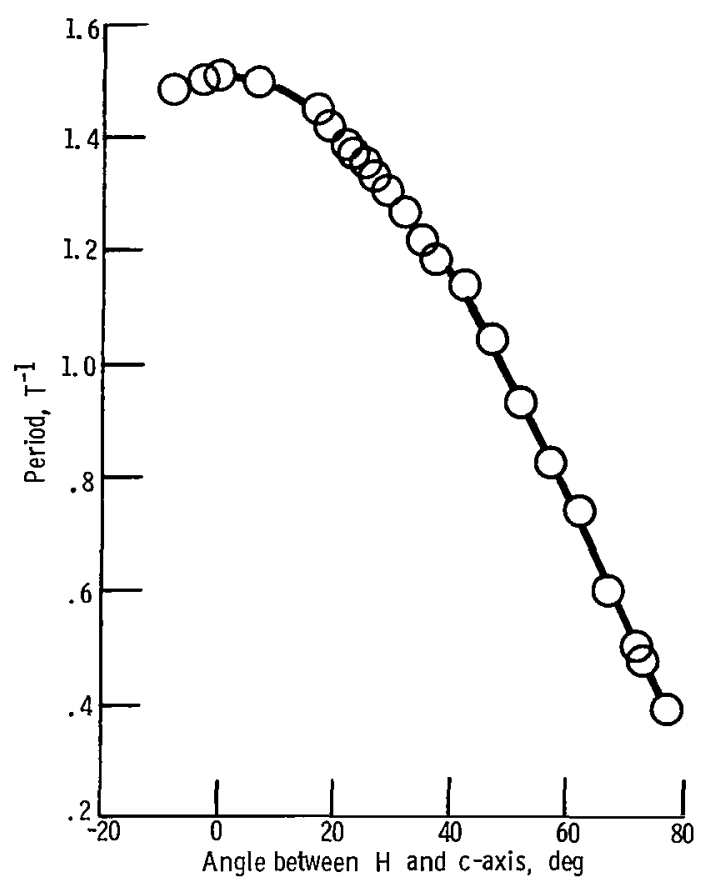

Figure 11. - Variation of period with angle.

CS -49783

(ref. 8) from de Haas - van Alphen measurements in pyrolytic graphite. The uncertainty in the period reported here is a direct result of the difficulty in determining the exact location of the small, lower field peaks. The field at which any peak occurred could be determined from the $\mathrm{X}-\mathrm{Y}$ recorder to within \pm 0.015 tesla. The period as a function of angle was determined by rotating the sample to the new position and sweeping the field. Consequently, the same uncertainty in period is associated with each point in the plot of figure 11.

The hole oscillations have superimposed on them a slowly varying envelope curve (fig. 12). The $1 / \mathrm{H}$ plot in figure 13 gives a period of $0.85 \pm 0.20$ tesla $^{-1}$. This oscilla-

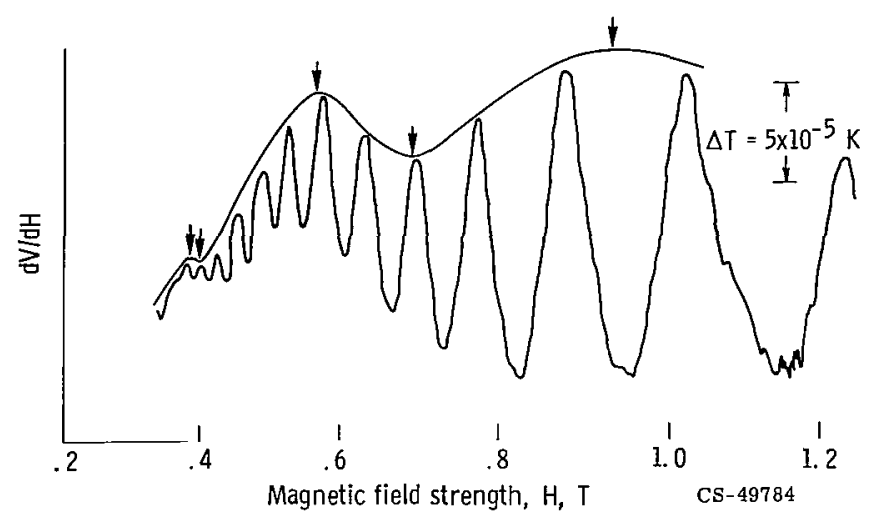

Figure 12. - Recorder trace of magnetothermal oscillations showing superimposed envelope curve and estimated locations of maximums and minimums. $\theta=0^{\circ}$ from $c$-axis; temperature, $1.32 \mathrm{~K}$. 


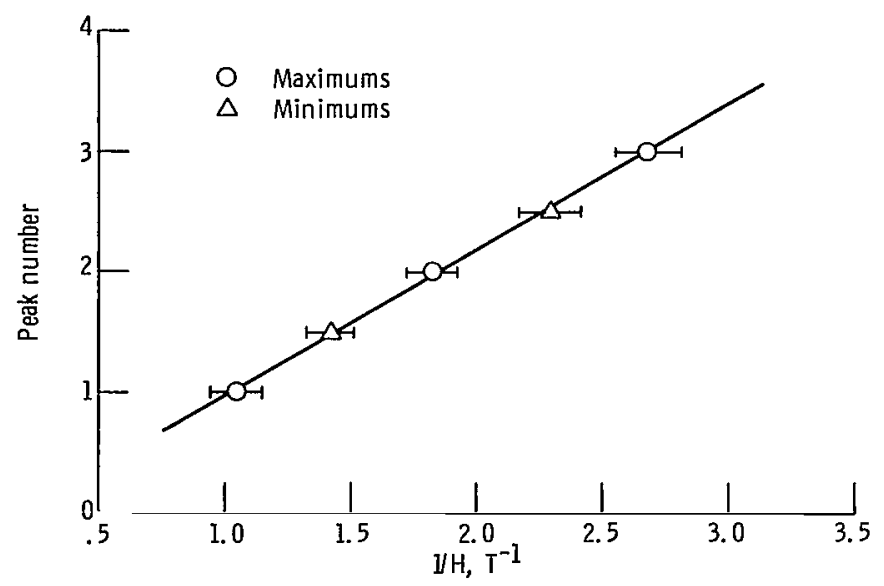

Figure 13. - Peak number as function of $1 / H$ for the long-period envelope oscillation. Period, $0.85 \pm 0.2$ tesla $^{-1}$.

tion is tentatively ascribed to the small pocket of minority electrons located at the points $\mathrm{H}, \mathrm{H}^{\prime}$ in the Brillouin zone (see fig. 2). The assignment is only a suggestion. Williamson et al. (ref. 8) found a minority period of $1.35_{ \pm} 0.01$ tes la $^{-1}$ in single crystal graphite, and $2.24 \pm 0.03$ tesla $^{-1}$ in pyrolytic graphite, for the same orientation. On the other hand, Soule (ref. 9) has reported the possible existance of three minority hole pockets in single crystal graphite which may be located around the majority hole pocket in "outrigger" fashion (see fig. 5). The period of this minority carrier oscillation varied from $0.58 \pm 0.1$ to $1.35_{ \pm} 0.3$ tesla $^{-1}$, depending on the amount of acceptor impurities introduced into the single crystal samples. The period of the superimposed oscillation given here could not be determined with very high precision. The presence of a remnant field in the superconducting solenoid prevented acquisition of data below about 0.35 tesla. Hence, only a few maximums and minimums could be observed, and the superposition of the shorter period hole oscillations made their location difficult to determine. The envelope oscillation was observable only at the lowest temperatures, and an attempt to investigate the low field region in another magnet failed because the same temperatures could not be reached.

The question of whether the observed long-period oscillation comes from a hole section or an electron section cannot be answered here. The sample has not yet been analyzed for impurities, since it was felt advisable to complete other measurements of its transport properties before cutting it. The presence of acceptor impurities would lower the period for a minority hole pocket, while the presence of donor impurities would lower the period for a minority electron pocket. A systematic investigation of the effects of such impurities would be necessary to establish the type of carrier. A spectrographic analysis of a similar piece of pyrolytic graphite has indicated the presence of $30 \mathrm{ppm}$ of aluminum, and only trace amounts $(<1 \mathrm{ppm})$ of boron. Aluminum would be a donor impurity and boron an acceptor. The presence of the aluminum favors the assignment of the 
oscillation to the minority electron pocket, since the samples were presumably prepared under the same conditions. The majority volumes are relatively unaffected by small impurity concentrations.

Figure 14 shows a recorder trace of $\mathrm{dT} / \mathrm{dH}$ as a function of $\mathrm{H}$ up to 8 tesla. The

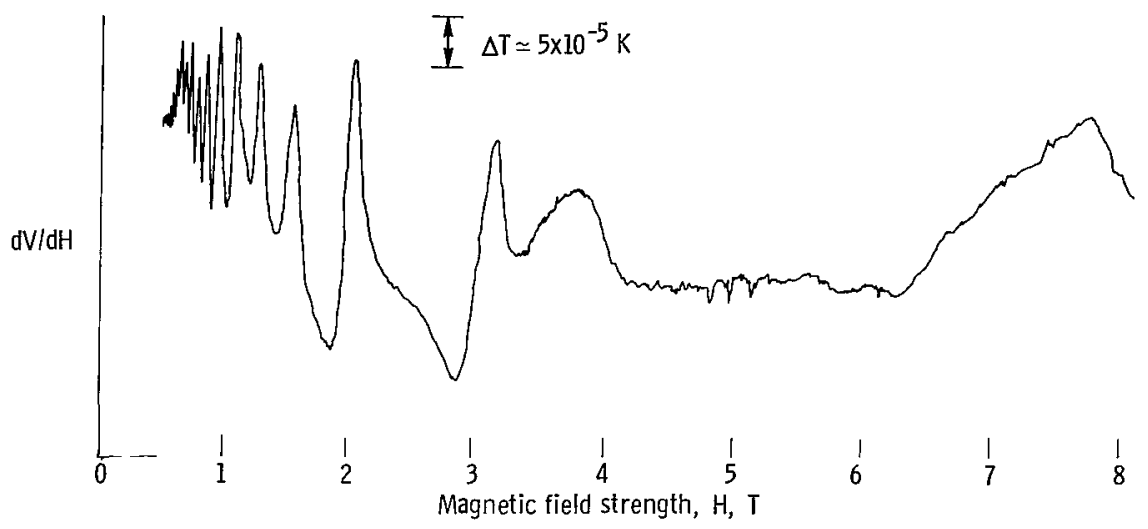

Figure 14. - Recorder trace of magnetothermal oscillations. $\theta=17^{\circ}$ from c-axis; temperature, $1.4 \mathrm{~K}$.

magnetic field is tilted $17^{\circ}$ from the c-axis. The figure clearly indicates how quickly the low quantum number regime is reached for the hole oscillations. The waveform is beginning to show some distortion by about 1.0 tesla, and is definitely nonsinusoidal for fields above 2 tesla. The frequency of the oscillations in figure 14 is $6.95 \pm 0.20$ tesla. Since the peaks in the figure actually correspond to maximums in $d V / d H$, where $V$ is the voltage across the thermometer, they are shifted from the maximums in $\mathrm{dT} / \mathrm{dH}$ by $180^{\circ}$. (Resistance as a function of temperature has a negative slope.) The condition for the occurrence of the $n^{\text {th }}$ maximum is therefore

$$
\frac{2 \pi \mathrm{f}}{\mathrm{H}}-2 \pi \gamma-\frac{\pi}{4}=2 \pi \mathrm{n}-\frac{\pi}{2}
$$

Table I lists the peak positions predicted by equation (10) and the fields at which they were actually observed. Equation (10) is not expected to be valid in the so-called quantum limit. The agreement between observed and predicted maximums is within experimental error for fields up to 2 tesla.

A plot of $\mathrm{n}$ as a function of $1 / \mathrm{H}$ for the field parallel to the c-axis is shown in figure 15. The integers were assigned to the peaks on the basis of the preceding discussion. The intercept may be used to calculate the quantity $\gamma$ in equation (10). From the figure, the intercept of the line is $\mathrm{n}=-0.37 \pm 0.10$. Inserting this in equation (10) gives $\gamma=0.5_{ \pm} 0.1$. 
TABLE I. - COM PARISON OF PREDICTED FIELD POSITIONS OF MAXIMUMS IN $\mathrm{dV} / \mathrm{dH}$ WITH OBSERVED FIELD POSITIONS

\begin{tabular}{|c|c|c|} 
Peak number, & \multicolumn{2}{|c|}{ Field position, $\mathrm{T}$} \\
\hline & Predicted & \multicolumn{1}{c|}{ Observed } \\
\hline 1 & 5.06 & $7.8_{ \pm 0.02}$ \\
2 & 2.93 & $3.2 \pm 0.02$ \\
3 & 2.06 & $2.05_{ \pm} 0.02$ \\
4 & 1.59 & $1.54_{ \pm} 0.02$ \\
5 & 1.29 & $1.27_{ \pm} 0.02$ \\
6 & 1.09 & $1.08_{ \pm} 0.02$ \\
7 & 9.43 & $9.30_{ \pm} 0.02$ \\
8 & 8.30 & $8.20_{ \pm} 0.02$ \\
9 & 7.42 & $7.50_{ \pm} 0.02$ \\
10 & 6.70 & $6.60_{ \pm} 0.02$ \\
11 & 6.12 & $6.10_{ \pm} 0.02$ \\
12 & 5.62 & $5.60_{ \pm} 0.02$ \\
\hline
\end{tabular}

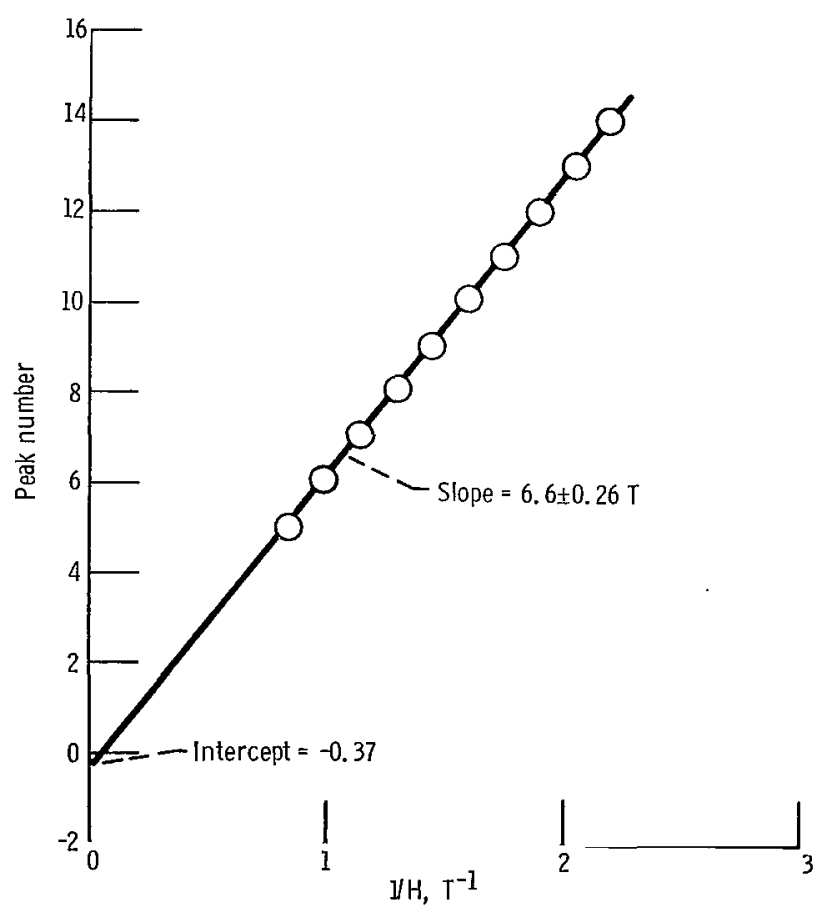

Figure 15. - Peak number as function of $\mathrm{yH}_{\mathrm{H}}$ for hole oscillations with $H$ along $c$-axis. 


\section{CONCLUSIONS}

The periodicity in $1 / \mathrm{H}$ of the oscillatory variations in temperature of the sample of pressure-annealed, pyrolytic graphite used in these experiments agrees well with that obtained for hole carriers by other workers in both single crystal and pyrolytic graphite. The measured value of the hole period for $H$ parallel to the $c$-axis was $0.151_{ \pm} 0.005$ tesla ${ }^{-1}$. From observations into the quantum limit, the phase factor $\gamma$ was found to be $0.5 \pm 0.10$.

A long-period oscillation was also observed which is tentatively ascribed to the minority electron pocket occurring at the corners of the Brillouin zone. The period of this oscillation was estimated to be $0.85 \pm 0.20$ tes $^{-1}$ and is not in good agreement with other published values for these carriers. One explanation for the different minority period observed in this study is the possible presence of donor impurities in the sample. An analysis of a similar piece of pyrolytic graphite has indicated the presence of $30 \mathrm{ppm}$ of aluminum and tends to corroborate this suspicion. Small traces of impurities could greatly affect the dimensions of the small pockets of minority carriers, while hardly affecting the majority carrier volumes.

Lewis Research Center,

National Aeronautics and Space Administration, Cleveland, Ohio, August 5, 1969, 129-02. 


\section{APPENDIX - SYMBOLS}

A symmetry point in hexagonal face of Brillouin zone

$A\left(E_{F}\right) \quad$ cross-sectional area of Fermi surface

$A_{n} \quad$ area enclosed by orbit of electrons in state labeled by quantum number $\mathbf{n}$

$A_{0}\left(E_{F}\right)$ extremal cross-sectional area of Fermi surface

a cross-sectional area of thermal path from sample to heat reservoir

$\mathrm{C}_{\mathrm{H}} \quad$ specific heat at constant magnetic field

c speed of light in vacuum

$c_{0} \quad$ twice the interplanar separation along the c-axis of graphite

$E_{F} \quad$ Fermi energy

$E_{n} \quad$ energy level of free electrons in a magnetic field

$\mathrm{E}_{1} \quad$ electronic energy band in graphite

$\mathrm{E}_{2} \quad$ electronic energy band in graphite

$\mathrm{E}_{3} \quad$ electronic energy band in graphite

f de Haas - van Alphen frequency of oscillations periodic in $1 / \mathrm{H}$

$\mathrm{g}$ effective spin-splitting factor for electrons in a magnetic field

$\mathrm{H} \quad$ magnetic field strength

$\mathrm{H}, \mathrm{H}^{\prime} \quad$ symmetry points along zone edges of hexagonal Brillouin zone

$\mathrm{h}, \mathrm{h} \quad$ Planck's constant (divided by $2 \pi$ )

$\mathrm{K}$ thermal conductivity of thermal path from sample to heat reservoir

$\mathrm{K}, \mathrm{K}^{\prime} \quad$ symmetry points at center of zone edges of hexagonal Brillouin zone

$\mathrm{k}_{\mathrm{B}} \quad$ Boltzmann constant

$\mathrm{k}_{\mathrm{o}} \quad$ value of $\mathrm{k}_{\mathrm{z}}$ at extremal area of Fermi surface

$\mathrm{k}_{\mathrm{x}} \mathrm{x}$-component of wave vector of electron

$\mathrm{k}_{\mathrm{y}} \quad \mathrm{y}$-component of wave vector of electron

$\mathrm{k}_{\mathrm{z}} \quad \mathrm{z}$-component of wave vector of electron

$\mathrm{L} \quad$ length of thermal path from sample to heat reservoir

M magnetization of a system of conduction electrons

$\mathbf{M}_{\text {osc }}$ oscillatory component of magnetization of system of conduction electrons 
$m_{0} \quad$ free electron mass

$m * \quad$ cyclotron effective mass of electron in a magnetic field

$\mathbf{n}$ quantum number labeling the energy level of an electron in a magnetic field

Q heat adsorbed or released by a thermodynamic system

$R_{300}$ sample resistance at room temperature

$R_{4.2}$ sample resistance in liquid helium

S entropy of thermodynamic system

$\mathrm{T}$ absolute temperature of thermodynamic system

$\mathrm{T}_{\mathrm{H}} \quad$ "magnetic temperature" defined by eq. (5b)

$\mathrm{U} \quad$ internal energy of thermodynamic system

$\mathrm{V}$ volume of system of conduction electrons

W work done by or on a thermodynamic system

$\mathrm{X} \quad$ Dingle temperature

$\gamma \quad$ phase factor in energy dispersion relation of electrons in a magnetic field

$\Gamma \quad$ symmetry point at center of hexagonal Brillouin zone

$\kappa \quad$ heat per second per degree transported along thermal path from sample to reservoir

$\tau_{\mathrm{D}} \quad$ average between collisions for an electron in a solid

$\omega^{*} \quad$ cyclotron frequency of electrons in a magnetic field 


\section{REFERENCES}

1. Kunzler, J. E.; Hsu, F. S. L.; and Boyle, W. S.: Magnetothermal Oscillations. The Oscillatory Dependence of Temperature on Magnetic Field. Phys. Rev., vol. 128, no. 3, Nov. 1, 1962, pp. 1084-1098.

2. LePage, J.; Garber, M.; and Blatt, F. J.: Magnetothermal Oscillations in Beryllium. Low Temperature Physics - LT9. Part B. J. G. Daunt, D. O. Edwards, F. J. Milford, and M. Yaqub, eds., Plenum Press, Inc., 1965, p. 799.

3. McCombe, B. D.; and Seidel, G. : Magnetothermal Oscillations in Semi-Metals. Low Temperature Physics - LT9. Part B. J. G. Daunt, D. O. Edwards, F. J. Milford, and M. Yaqub, eds., Plenum Press, Inc., 1965, p. 794.

4. McCombe, B. D.; Broshar, W.; and Seidel, G.: Magnetic Oscillations in Beryllium. Bull. Am. Phys. Soc., vol. 11, no. 1, Jan. 1966, p. 91.

5. McCombe, B.; and Seidel, G.: Magnetothermal Oscillations and Spin Splitting in Bismuth and Antimony. Phys. Rev., vol. 155, no. 3, Mar. 15, 1967, pp. 633-641.

6. Klein, C. A.; Straub, W. D.; and Diefendorf, R. J.: Evidence of Single-Crystal Characteristics of Highly Annealed Pyrolytic Graphite. Phys. Rev., vol. 125, no. 2, Jan. 15, 1962 , pp. 468-470.

7. Klein, Claude A.: Electrical Properties of Pyrolytic Graphites. Rev. Mod. Phys., vol. 34 , no. 1 , Jan. 1962 , pp. 56-79.

8. Williamson, S. J.; Foner, S.; and Dresselhaus, M. S.: de Haas-van Alphen Effect in Pyrolytic and Single-Crystal Graphite. Phys. Rev., vol. 140A, no. 4, Nov. 15, 1962, pp. 1429-1447.

9. Soule, D. E. : Change in Fermi Surfaces of Graphite by Dilute Acceptor Doping.

IBM J. Res. Dev., vol. 8, no. 3, July 1964, pp. 268-273.

10. Soule, D. E.; McClure, J. W. ; and Smith, L. B.: Study of the Shubnikov-de Haas Effect Determination of the Fermi Surfaces in Graphite. Phys. Rev., vol. 134A, no. 2, Apr. 20, 1964, pp. 453-470.

11. Slonczewski, J. C.; and Weiss, P. R.: Band Structure of Graphite. Phys. Rev., vol. 109, no. 2, Jan. 15, 1958, pp. 272-279.

12. Lifshitz, I. M.; and Kosevich, A. M.: Theory of Magnetic Susceptibility in Metals at Low Temperatures. Soviet Phys.-JETP, vol. 2, no. 4, July 1956, pp. 636-645.

13. Cohen, M. H. ; and Blount, E. I. : The g-factor and de Haas-van Alphen Effect of Electrons in Bismuth. Phil. Mag., vol. 5, no. 50, Feb. 1960, pp. 115-126. 
14. Dingle, R. B. : Some Magnetic Properties of Metals. $\Pi$. The Influence of Collisions on the Magnetic Behaviour of Large Systems. Proc. Roy. Soc. (London), Ser. A, vol. 211, no. 1107, Mar. 20, 1952, pp. 517-525.

15. Williamson, S. J.; Foner, S.; and Smith, R. A. : Influence of Landau Level Broadening on the de Haas-van Alphen Effect. Phys. Rev., vol. 136A, no. 4, Nov. 16, 1964, pp. 1065-1067.

16. Onsager, L.: Interpretation of the de Haas-van Alphen Effect. Phil. Mag., vol. 43, no. 344 , Sept. 1952 , pp. 1006-1008.

17. Chambers, R. G.: The Wave Function of a Block Electron in a Magnetic Field. Proc. Phys. Soc. (London), vol. 89, pt. 3, Nov. 1966, pp. 695-710.

18. McClure, J. W.: Theory of Diamagnetism of Graphite. Phys. Rev., vol. 119, no. 2 , July 15,1960 , pp. 606-613. 